\title{
Factors influencing haze formation and corneal flattening, and the impact of haze on visual acuity after conventional collagen cross-linking: a 12-month retrospective study
}

\author{
Anita Csorba ${ }^{1 *} \mathbb{D}$, Kinga Kránitz ${ }^{1}$, Péter Dormán ${ }^{1}$, Andrea Popper-Sachetti ${ }^{1}$, Huba Kiss ${ }^{1}$, Irén Szalai ${ }^{1}$ and \\ Zoltán Zsolt Nagy ${ }^{1,2}$
}

\begin{abstract}
Background: Our aim was to determine associations of pachymetry, keratometry, and their changes with haze formation and corneal flattening after collagen cross-linking, and to analyse the relationship between postoperative haze and visual outcome.

Methods: Retrospective analysis was performed on 47 eyes of 47 patients with keratoconus using the Pentacam HR Scheimpflug camera before and 1, 3, 6 and 12 months after cross-linking. Corneal backscattered light values in grey scale unit were recorded in the anterior, center and posterior corneal layers and in four concentric rings. Surface area- and thickness-corrected grey scale unit values were assessed with an additional calculation. Friedman test with post hoc Wilcoxon signed-rank test was used to analyse changes in visual acuity, pachymetry, keratometry and densitometry. Spearman's rank correlation test was used to detect correlations of haze formation and corneal flattening with pachymetry, keratometry and their postoperative change. Generalized estimating equations analysis was used to investigate the influence of densitometry values on postoperative visual acuity after controlling for the effect of preoperative keratometry.
\end{abstract}

Results: One year after treatment, significant flattening was observed in maximum and mean keratometry readings $(p<0.001)$. Significantly increased densitometry values were observed in three central rings compared to baseline (post hoc $p<0.0125$ ). According to receiver operating characteristic curve, densitometry value of the anterior layer of $0-2 \mathrm{~mm}$ ring was the most characteristic parameter of densitometry changes after cross-linking (area under the curve $=0.936)$. Changes in haze significantly correlated with preoperative maximum keratometry $(R=0.303, p=$ $0.038)$ and with the changes in maximum keratometry $(R=-0.412, p=0.004)$. Changes in maximum keratometry correlated with preoperative maximum keratometry $(R=-0.302, p=0.038)$. Postoperative haze had a significant impact on uncorrected and best corrected distance visual acuity ( $\beta$ coefficient $=0.006, p=0.041$ and $\beta$ coefficient $=$ $0.003, p=0.039$, respectively).

\footnotetext{
* Correspondence: csorba.anita@semmelweis-univ.hu

'Department of Ophthalmology, Semmelweis University, Budapest, Hungary

Full list of author information is available at the end of the article
} 
Conclusions: Our findings indicate that in more advanced keratoconus more significant corneal flattening effect parallel with haze formation can be observed after cross-linking. Despite significant reduction of keratometry, postoperative corneal haze may limit final visual acuity.

Keywords: Keratoconus, Conventional cross-linking, Densitometry, Corneal haze, Visual outcome

\section{Background}

Keratoconus is a bilateral, asymmetric corneal disorder, which leads to progressive protrusion and thinning of the cornea including irregular astigmatism and deterioration of visual acuity [1]. The only effective treatment which can prevent the progression is the minimalinvasive corneal collagen cross-linking (CXL) therapy. Combination of UV-A light and photosensitising riboflavin produces reactive oxigen species, which induces crosslinks between collagen fibrils. Photopolymerization of collagen monomers increases corneal rigidity after treatment [2]. Results of the first clinical study performing CXL treatment on human corneas were published by Wollensak et al. in 2003 [3]. Since then, several longterm clinical studies [4-9] have shown promising clinical outcomes in terms of safety and efficacy.

Corneal haze induces increased backward corneal light scattering, which can be measured by Pentacam HR [10]. Since 'Cornea Densito' software is available, the degree and location of corneal opacities can be quantified objectively in concentric rings and in different depth layers of the cornea. Several studies have demonstrated increased densitometry values after CXL in keratoconus patients [11-14], mainly in the anterior and middle layers and central rings. Previous studies have already evaluated correlations between densitometry values and postoperative outcomes $[15,16]$, however no preoperative predictive factors of postoperative corneal haze formation have been yet identified after conventional CXL.

The purpose of the current study was to characterize changes in visual acuity and of corneal haze, curvature, and thickness during a one-year follow-up period after CXL treatment in keratoconus patients. Besides, we aimed to identify the relationship of pachymetry, keratometry, and their postoperative changes with corneal flattening and haze formation, and analyse the associations between postoperative haze and visual outcome.

\section{Methods}

\section{Patients and study design}

This retrospective study was conducted at the Department of Ophthalmology, Semmelweis University with the approval of the Semmelweis University Regional and Institutional Committee of Sciences and Research Ethics. Each participant was informed about the treatments, and written consent was obtained from all patients. All examinations were carried out in accordance with the tenets of the Declaration of Helsinki.

Fourty-seven patients with progressive keratoconus underwent conventional CXL between 2017 and 2018 were enrolled. The surgical intervention was indicated when ectatic progression i.e. increase of maximum keratometry values ( $\geq 1.0$ diopter in 1 year) and/or loss of vision (loss of $\geq$ two lines of best corrected distance visual acuity in 1 year) was decetected [17]. Patients with age under 18 years, preoperative corneal scarring, previous history of corneal hydrops, prior keratitis, history of any eye injury or corneal thickness below $400 \mu \mathrm{m}$ were excluded. All pre-, and postoperative data were collected and analysed retrospectively.

\section{Surgical procedure}

Conventional corneal CXL was performed by the same surgeon (Z.Zs.N.) according to Dresden protocol [3]. Oxybuprocaine eye drops (Benoxi ${ }^{\circ}$, Unimed Pharma, Bratislava, Slovakia) were instilled preoperatively. Following mechanical removal of epithelial layer in a central diameter of $8 \mathrm{~mm}, 0.1 \%$ riboflavin droplets (MedioHaus Medizinprodukte $\mathrm{GmbH}$, Rostock, Germany) were administered topically in every 2 min for $30 \mathrm{~min}$. The cornea was then exposed to UV-A light from a 1-cm distance with a wavelength of $370 \pm 5 \mathrm{~nm}$ (CSO Vega CMB $\mathrm{X}$ Linker, CSO Scandicci, Firenze, Italy) at an irradiance of $3 \mathrm{~mW} / \mathrm{cm}^{2}$ intensity for the next $30 \mathrm{~min}$ with continued instillation of riboflavin in every $2 \mathrm{~min}$. Finally, topical antibiotic drops (5 $\mathrm{mg} / \mathrm{ml}$ levofloxacin) were instilled and a bandage was placed on. In the early postoperative period, all patients were examined the day after the surgery, when the bandage was removed, and 1 week later after it, when complete corneal reepithelization was confirmed. Antibiotic drops $(5 \mathrm{mg} / \mathrm{ml}$ levofloxacin) were instilled five times daily during the first week postoperatively. After complete reepithelizaiton, in the following one month, topical corticosteroid (1 $\mathrm{mg} / \mathrm{ml}$ fluorometholone) was administered 4 times per day.

\section{Examinations}

Ophthalmological examinations included uncorrected (UCDVA) and best corrected distance visual acuity (BCDVA) assessment (measured with Snellen charts and converted to $\log$ MAR values) with recording spherical equivalent (SEQ) followed by slit-lamp biomicroscopy 
and Scheimpflug imaging performed preoperatively and at 1, 3, 6 and 12 months after CXL procedure. Pentacam HR Scheimpflug rotating camera (Pentacam HR, Oculus Optikgeräte $\mathrm{GmbH}$, Wetzlar, Germany) was used to measure the thinnest corneal thickness (ThCT), the maximum keratometry values $\left(\mathrm{K}_{\max }\right)$, mean keratometry values $\left(\mathrm{K}_{\text {mean }}\right)$ and the corneal densitometry values. Only measurements with good image quality - "OK” was shown in quality specification window - were accepted.

Cornea Densito module shows a chart with the average densitometry values of the cornea in grey scale unit (GSU; 0-100 light scattering; 0: maximal trasparency/ optically clear cornea; 100: minimal transparency/total corneal opacification) in four concentric rings $(0-2 \mathrm{~mm}$; 2-6 $\mathrm{mm} ; 6-10 \mathrm{~mm} ; 10-12 \mathrm{~mm}$ ) and in three different layers. The "Anterior" layer represents the front $120 \mu \mathrm{m}$, while the "Posterior" refers the last $60 \mu \mathrm{m}$ of corneal thickness from epithelial to endothelial layer. The "Center" layer gives the values between these two layers, thus, the thickness of this layer is variable in every patients. "Total" layer represents the average optical density of the full corneal thickness. Additionally, since different rings have different surface areas and thicknesses, raw densitometry data in GSU were converted to GSU/cubic millimeters $\left(\mathrm{GSU} / \mathrm{mm}^{3}\right)$, as previously published by Nemeth et. al [13]. First, we received values in GSU/ $\mathrm{mm}^{2}$ with dividing raw GSU data of each concentric ring by its area $\left(3.141 \mathrm{~mm}^{2}\right.$ for ring $0-2 \mathrm{~mm} ; 25.132 \mathrm{~mm}^{2}$ for ring 2-6 mm; $50.265 \mathrm{~mm}^{2}$ for ring $6-10 \mathrm{~mm} ; 34.557$ $\mathrm{mm}^{2}$ for ring $10-12 \mathrm{~mm}$ and $78.539 \mathrm{~mm}^{2}$ for total diameter). Then values of different rings in GSU $/ \mathrm{mm}^{2}$ were divided by the thickness of the appropriate layer in $\mathrm{mm}(0.12 \mathrm{~mm}$ for the anterior layer and 0.06 for the posterior layer). The thickness of the center layer was calculated by substracting 120 and $60 \mu \mathrm{m}$ from total thickness which was recorded from "Pachy apex" window (the $\mathrm{X}$ and $\mathrm{Y}$ coordinates of this point are 0.00 ) and then it was converted to $\mathrm{mm}$. With this additional calculation we got surface area- and thickness-corrected densitometry values (cGSU) in GSU/mm ${ }^{3}$.

\section{Statistical analysis}

The statistical analysis was performed using $\mathrm{IBM}^{\circ} \mathrm{SPSS}^{\circ}$ Statistics for Windows, version 25.0 (IBM Corp., Armonk, N.Y., USA). Kolmogorov-Smirnov test was used to assess the normality of the variables. Based on the results of normality test, the changes in UCDVA, BCDVA, SEQ, keratometry, pachymetry and densitometry were evaluated using nonparametric Friedman test. Where Friedman test resulted in statistical significance, post hoc pairwise comparison was implemented using Wilcoxon signed-rank test for comparison of each time point to the baseline (i.e. baseline vs. 1 month, baseline vs. 3 months, baseline vs. 6 months and baseline vs. 12 months). As these four analyses were conducted for each repeated testing procedure, Bonferroni-adjusted significance level was applied for each comparison resulting in a significance level at $p<0.0125$. Receiver operating characteristic (ROC) curve was plotted to determine the most characteristic densitometry data of corneal changes at 12-month visit. Comparison of ROC curves was performed to test the statistical significance of the difference between the areas under the curves (AUC) with the method of DeLong et al. using MedCalc ${ }^{\circ}$, version 19.4.1. (MedCalc Software Ltd., Ostend, Belgium) [18]. Spearman's rank correlation test was used to find relationships between pachymetry, keratometry, and their postoperative changes and in haze formation and corneal flattening. The associations of postoperative visual acuity (dependent variable of interest) with the densitometry values (independent variable) 1 year after surgery was assessed with generalized estimating equations (GEE). Preoperative maximum keratometry was incorporated as a covariate in the regression model to adjust for potentional cofounding. The level of significance was considered at $p<0.05$ in all analysis, except the post hoc Wilcoxon test.

\section{Results}

Fourty-seven eyes of 47 patients including 35 male and 12 female subjects with keratoconus underwent conventional CXL therapy were involved in this study. The mean age was $26.72 \pm 6.03$ (range, 18-38) years before CXL. All involved subjects were Caucasian. The cohort comprised 21 eyes (44.7\%) in stage I, 19 eyes (40.4\%) in stage II and 7 eyes (14.9\%) in stage III according to Amsler-Krumeich classification of keratoconus severity [19]. No ophthalmological complications developed due to the CXL surgery during the follow-up period.

Friedman test revealed statistically significant changes in SEQ postoperatively $(p<0.001)$. Post hoc evaluation showed significant improvement 6 and 12 months after CXL ( $p<0.001$ and $p<0.002$, respectively). According to Friedman test, statistically significant changes were found both in UCDVA and in BCDVA after CXL (both $p<0.001)$. Regarding UCDVA, significant difference was found at 1 month compared to baseline (post hoc $p=$ 0.005). At 1-, 3-, and 6-month visit, BCDVA was lower than preoperatively (post hoc $p>0.0125$ ), but the difference was statistically significant only also at the first postoperative month, as revealed by post hoc Wilcoxon signed-rank test (post hoc $p=0.005$ ). At 12 months, improvement in was not significant compared to the baseline value neither in UCDVA, nor in BCDVA (post hoc $p>0.0125$ ).

There were significant changes both in $\mathrm{K}_{\max }$ and $\mathrm{K}_{\text {mean }}$ values and in ThCT values postoperatively, as determined by Friedman test (both $p$ values $<0.001$ ). Post hoc 
analysis revealed that at 1 month there was a significant increase in $\mathrm{K}_{\max }$ value from preoperative $56.41 \pm 5.41 \mathrm{D}$ to $57.15 \pm 5.74 \mathrm{D}$ (post hoc $p<0.001$ ), after which keratometry began to decrease. One year after CXL, $K_{\max }$ readings decreased significantly to $55.35 \pm 5.18 \mathrm{D}$ compared to baseline (post hoc $p<0.001$ ). Change in $\mathrm{K}_{\text {mean }}$ showed similar tendency with significant changes at 3, 6 and 12 months (post hoc $p<0.001$ ). Average decrease was $-1.05 \pm 1.13 \mathrm{D}$ in $\mathrm{K}_{\max }$ value $\left(\Delta \mathrm{K}_{\max }\right)$ and it was $-0.71 \pm 0.78 \mathrm{D}$ in $\mathrm{K}_{\text {mean }}$ during follow-up period. Compared to the preoperative value, ThCT decreased significantly in all postoperative month (all post hoc $p$ values $<0.001)$. One year after treatment, ThCT decreased by $-15.5 \pm 15.19 \mu \mathrm{m}$. UCDVA, BCDVA, SEQ, pachymetry and keratometry values at various times are listed in Table 1.

Table 2 shows the mean cGSU values at different follow up visits. Statistically significant changes were found in densitometry values of anterior, central and total layer of $0-2 \mathrm{~mm}, 2-6 \mathrm{~mm}$ and $6-10 \mathrm{~mm}$ ring after CXL according to Friedman test (all $p$ values $<0.001$ ). Upon further testing using the Wilcoxon test, regarding the concentric rings of the cornea, in $0-2 \mathrm{~mm}$ and in $2-6$ $\mathrm{mm}$ rings, cGSU values were significantly increased at all postoperative visits in anterior, center and total layers (all post hoc $p$ values $<0.0125$ ). In $6-10 \mathrm{~mm}$ ring, significantly increased cGSU was found in the anterior layer at 1 and 3 months (both post hoc $p<0.001$ ) and in center and total layers at all follow-up visits (post hoc $p<0.0125)$. There was a peak in densitometry values 3 months after CXL in the most central zone and after 1 month in the $2-6 \mathrm{~mm}$ and $6-10 \mathrm{~mm}$ rings. After the peak, densitometry values decreased. Considering the different corneal layers, Friedman test showed significant changes in total densitometry of anterior, center and total layers (all $p<0.001$ ). Post hoc analysis revealed that densitometry values of these layers were significantly increased at all postoperative visits (all post hoc $p<0.001$ ) except the total densitometry of center layer at 12 months (post hoc $p=0.079$ ). Densitometry values in the remaining zones did not change significantly after CXL (all post hoc $p>0.0125$ ) (Table 2).

Figure 1 shows the results of ROC curve analysis for corrected densitometry values. For ROC analysis, significantly increased corrected densitometry data at 12 months were used from the central rings mostly affected by CXL (anterior, center and total layer of $0-2 \mathrm{~mm}$ and of $2-6$ $\mathrm{mm}$ rings). According to ROC curve, at postoperative 12 months, the corrected densitometry in the anterior layer of the $0-2 \mathrm{~mm}$ ring (cGSU-0-2A) was the most characteristic parameter of corneal densitometry changes after CXL (AUC $=0.936$. AUC of cGSU-0-2A was statistically significant compared to other AUCs (cGSU-0-2C, $p<0.001$; cGSU-0-2T, $p<0.001$; cGSU-2-6A, $p<0.001$; cGSU-2-6C, $p<0.001$ and cGSU-2-6T, $p<0.001$, respectively). Therefore, the data of cGSU-0-2A region were hereinafter used to examine the relationship between preoperative data and densitometry values. Average increase in densitometry of this region was $21.35 \pm 14.69 \mathrm{GSU} / \mathrm{mm}^{3}$ as compared to the baseline (Fig. 1).

Considering the relationship between the changes in densitometry ( $\triangle \mathrm{cGSU}-0-2 \mathrm{~A})$ and the preoperative parameters, one year after $\mathrm{CXL}$, the changes in haze in this region was statistically correlated with preoperative $\mathrm{K}_{\max }$ $(R=0.303, p=0.038) \quad$ (Fig. 2). At postoperative 12 months, $\Delta$ cGSU-0-2A was moderately correlated with $\Delta \mathrm{K}_{\max }(R=-0.412, p=0.004)$ (Fig. 3). No correlation was observed between $\Delta$ cGSU-0-2A and preoperative ThCT $(R=-0.022, p=0.885)$ and changes in thinnest pachymetry $(\Delta \mathrm{ThCT})(R=-0.27, p=0.066)$. Age did not have any effect neither on cGSU-0-2A at postoperative 12 months $(R=0.177, p=0.233)$ nor on $\Delta$ cGSU-0-2A $(R=0.097, p=0.514)$. The analysis of the influence of preoperative values on corneal flattening showed significant negative correlation between $\Delta \mathrm{K}_{\max }$ and preoperative $K_{\max }$ readings $(R=-0.302, p=0.038)$ however $\Delta \mathrm{K}_{\max }$ did not correlated with preoperative ThCT $(R=$ 0.094, $p=0.53$ ) (Fig. 4).

GEE analysis showed that postoperative densitometry (cGSU-0-2A) has a significant effect both on

Table 1 Uncorrected- and best corrected distance visual acuity (UCDVA and BCDVA, logMAR), spherical equivalent (SEQ, D), thinnest corneal pachymetry $(T h C T, \mu m)$, maximum and mean keratometry $\left(K_{\max }\right.$ and $\left.K_{\text {mean }}, D\right)$ at different visits

\begin{tabular}{|c|c|c|c|c|c|}
\hline \multirow[t]{2}{*}{ Variables } & \multirow[t]{2}{*}{ Preoperative } & \multicolumn{4}{|l|}{ Postoperative } \\
\hline & & 1 months & 3 months & 6 months & 12 months \\
\hline BCDVA (logMAR) & $0.16 \pm 0.2$ & $0.23 \pm 0.21^{\dagger}$ & $0.18 \pm 0.19$ & $0.18 \pm 0.18$ & $0.13 \pm 0.2$ \\
\hline UCDVA (logMAR) & $0.56 \pm 0.38$ & $0.64 \pm 0.35^{\dagger}$ & $0.56 \pm 0.35$ & $0.55 \pm 0.34$ & $0.51 \pm 0.32$ \\
\hline SEQ (D) & $-3.77 \pm 3.74$ & $-2.97 \pm 2.61$ & $-2.95 \pm 2.65$ & $-2.65 \pm 2.65^{\dagger}$ & $-2.59 \pm 2.58^{\dagger}$ \\
\hline $\mathrm{ThCT}(\mu \mathrm{m})$ & $476.49 \pm 34.57$ & $444.3 \pm 38.88^{\dagger}$ & $451.39 \pm 37.75^{\dagger}$ & $455.11 \pm 36.52^{\dagger}$ & $460.94 \pm 35.74^{\dagger}$ \\
\hline $\mathrm{K}_{\max }(\mathrm{D})$ & $56.41 \pm 5.41$ & $57.15 \pm 5.74^{\dagger}$ & $56.01 \pm 6.03$ & $55.75 \pm 5.67$ & $55.35 \pm 5.18^{\dagger}$ \\
\hline$K_{\text {mean }}(D)$ & $47.47 \pm 3.49$ & $47.54 \pm 3.87$ & $46.93 \pm 3.64^{\dagger}$ & $46.81 \pm 3.61^{\dagger}$ & $46.76 \pm 3.31^{\dagger}$ \\
\hline
\end{tabular}

Data are shown as mean \pm standard deviation

${ }^{\dagger}$ Statistically significant difference compared to baseline (Bonferroni-adjusted post hoc $p$-value $<0.0125$ ) 
Table 2 Corrected densitometry values in grey scale unit per cubic millimeter (GSU/mm²) at all postoperative months

\begin{tabular}{|c|c|c|c|c|c|c|c|c|}
\hline \multirow[t]{2}{*}{ Variables } & & \multirow[t]{2}{*}{ Preoperative } & \multicolumn{4}{|l|}{ Postoperative } & \multirow[t]{2}{*}{$p^{*}$} & \multirow[t]{2}{*}{$p^{* *}$} \\
\hline & & & 1 months & 3 months & 6 months & 12 months & & \\
\hline \multirow[t]{5}{*}{ Anterior } & $0-2 \mathrm{~mm}$ & $79.29 \pm 6.75$ & $107.45 \pm 17.86 \dagger$ & $113.55 \pm 21.8+$ & $104.27 \pm 16.86 \dagger$ & $100.64 \pm 14.06+$ & $<0.001$ & $<0.001$ \\
\hline & $2-6 \mathrm{~mm}$ & $8.45 \pm 0.66$ & $11.07 \pm 1.35 t$ & $10.71 \pm 1.12 \dagger$ & $9.76 \pm 1.13 \dagger$ & $9.4 \pm 0.84 \dagger$ & $<0.001$ & $<0.001$ \\
\hline & $6-10 \mathrm{~mm}$ & $3.39 \pm 0.37$ & $3.83 \pm 0.63 \dagger$ & $3.56 \pm 0.41 \dagger$ & $3.42 \pm 0.37$ & $3.42 \pm 0.36$ & $<0.001$ & $=0.17$ \\
\hline & $10-12 \mathrm{~mm}$ & $6.54 \pm 1.87$ & $6.23 \pm 2.05$ & $6.27 \pm 2.09$ & $6.27 \pm 1.74$ & $6.45 \pm 2.23$ & $=0.895$ & \\
\hline & Total & $2.63 \pm 0.24$ & $3.17 \pm 0.37 \dagger$ & $3.13 \pm 0.36+$ & $2.93 \pm 0.33 \dagger$ & $2.87 \pm 0.31 \dagger$ & $<0.001$ & $<0.001$ \\
\hline \multirow[t]{5}{*}{ Center } & $0-2 \mathrm{~mm}$ & $18.74 \pm 2.41$ & $25.49 \pm 5.93 \dagger$ & $26.17 \pm 8.23+$ & $22.98 \pm 5.23 \dagger$ & $22.26 \pm 9.68 \dagger$ & $<0.001$ & $<0.001$ \\
\hline & $2-6 \mathrm{~mm}$ & $1.98 \pm 0.27$ & $2.63 \pm 0.53 \dagger$ & $2.49 \pm 0.58 \dagger$ & $2.24 \pm 0.36 t$ & $2.12 \pm 0.33 \dagger$ & $<0.001$ & $<0.001$ \\
\hline & $6-10 \mathrm{~mm}$ & $0.89 \pm 0.13$ & $1.07 \pm 0.27 \dagger$ & $1.0 \pm 0.18 \dagger$ & $0.96 \pm 1.16 \dagger$ & $0.93 \pm 0.15 \dagger$ & $<0.001$ & $=0.003$ \\
\hline & $10-12 \mathrm{~mm}$ & $1.83 \pm 0.53$ & $1.86 \pm 0.46$ & $1.92 \pm 0.64$ & $1.94 \pm 0.56$ & $1.85 \pm 0.5$ & $=0.119$ & \\
\hline & Total & $0.66 \pm 0.08$ & $0.82 \pm 0.18 \dagger$ & $0.79 \pm 0.17 \dagger$ & $0.74 \pm 0.13 \dagger$ & $0.66 \pm 0.08$ & $<0.001$ & $=0.079$ \\
\hline \multirow[t]{5}{*}{ Posterior } & $0-2 \mathrm{~mm}$ & $67.88 \pm 9.33$ & $68.98 \pm 9.39$ & $71.65 \pm 13.7$ & $66.73 \pm 9.49$ & $67.68 \pm 9.03$ & $=0.418$ & \\
\hline & $2-6 \mathrm{~mm}$ & $8.50 \pm 0.78$ & $10.09 \pm 9.82$ & $8.61 \pm 0.74$ & $8.24 \pm 0.82$ & $8.27 \pm 0.64$ & $=0.032$ & $=0.039$ \\
\hline & $6-10 \mathrm{~mm}$ & $4.24 \pm 0.63$ & $4.32 \pm 0.78$ & $4.23 \pm 0.59$ & $4.12 \pm 0.51$ & $4.16 \pm 0.49$ & $=0.439$ & \\
\hline & $10-12 \mathrm{~mm}$ & $8.43 \pm 0.94$ & $8.37 \pm 1.99$ & $8.2 \pm 1.89$ & $8.43 \pm 1.83$ & $8.61 \pm 1.96$ & $=0.451$ & \\
\hline & Total & $2.86 \pm 0.33$ & $2.89 \pm 0.38$ & $92.87 \pm 0.32$ & $2.8 \pm 0.29$ & $2.83 \pm 0.26$ & $=0.659$ & \\
\hline \multirow[t]{5}{*}{ Total } & $0-2 \mathrm{~mm}$ & $13.25 \pm 1.34$ & $17.34 \pm 3.92 \dagger$ & $18.3 \pm 3.92 \dagger$ & $16.54 \pm 2.81 \dagger$ & $15.94 \pm 3.02 \dagger$ & $<0.001$ & $<0.001$ \\
\hline & $2-6 \mathrm{~mm}$ & $1.46 \pm 0.14$ & $1.89 \pm 0.28+$ & $1.88 \pm 0.28+$ & $1.65 \pm 0.21 \dagger$ & $1.58 \pm 0.16 \dagger$ & $<0.001$ & $<0.001$ \\
\hline & $6-10 \mathrm{~mm}$ & $0.64 \pm 0.07$ & $0.74 \pm 0.15 \dagger$ & $0.69 \pm 0.08+$ & $0.67 \pm 0.08 \dagger$ & $0.66 \pm 0.07 \dagger$ & $<0.001$ & $=0.007$ \\
\hline & $10-12 \mathrm{~mm}$ & $1.27 \pm 0.33$ & $1.33 \pm 0.42$ & $1.29 \pm 0.38$ & $1.29 \pm 0.33$ & $1.32 \pm 0.38$ & $=0.473$ & \\
\hline & Total & $0.47 \pm 0.05$ & $0.57 \pm 0.1 \dagger$ & $0.56 \pm 0.08 \dagger$ & $0.52 \pm 0.07 \dagger$ & $0.51 \pm 0.06 \dagger$ & $<0.001$ & $<0.001$ \\
\hline
\end{tabular}

Data are shown as mean \pm standard deviation

${ }^{\dagger}$ Statistically significant difference compared to baseline (Bonferroni-adjusted $p$-value $<0.0125$ )

${ }^{*} p$-values of Friedman tests

**Post hoc $p$-value of 12-month data compared to baseline

postoperative UCDVA $(\beta$ coefficient $=0.006, p=0.041)$ and postoperative BCDVA $(\beta$ coefficient $=0.003, p=$ 0.039 ) after adjusted for the effect preoperative $K_{\max }$ readings $(\beta$ coefficient $=0.026, p<0.001$ and $\beta$ coefficient $=0.018, p<0.001$, respectively) (Table 3 ).

\section{Discussion}

Herein in the present study, classical corneal densitometry alterations measured by Cornea Densito software of Pentacam HR after conventional CXL therapy in keratoconus patients during 1-year follow-up were reported. Beside this, the impact of keratometric and pachymetric values on haze formation and corneal flattening were also assessed.

The highly organized structure of corneal tissue is an important factor of corneal transparency [20]. However, corneal collagen cross-linking is considered as a safe technique to stabilize the cornea in progressive keratoconus, it may induce haze formation. Previous confocal microscopic studies [21, 22] found various cellular changes after CXL, which might modify corneal transparency and associated with densitometry changes. In the early postoperative months, keratocyte loss due to apoptosis and edematous, hyper-reflective extracellular stroma can be observed. These alterations may last for 6 months, but generally disappears after 12 months postoperatively, which is followed by resolution of the haze [22].

In our study, we found initial worsening of UCDVA and BCDVA at the first postoperative month, which was followed by an improvement; however, the difference was not statistically significant compared to the preoperative values at 12 months. Changes in $\mathrm{K}_{\text {mean }}$ and $\mathrm{K}_{\max }$ readings showed similar trend with steepening at 1 month, after which they began to stabilize and showed significant flattening one year after treatment. Densitometry values were peaked between the first and the third postoperative months, after they began to decrease. Presumably, our findigs are consistent with the aforementioned cellular changes, i.e. CXL-induced stromal modifications cause higher keratometry and densitometry values and worsening of visual acuity in early postoperative months, but later with remodelling process these parameters are stabilized.

In this detailed analysis of corneal densitometry, increased densitometry values were found after CXL in the 


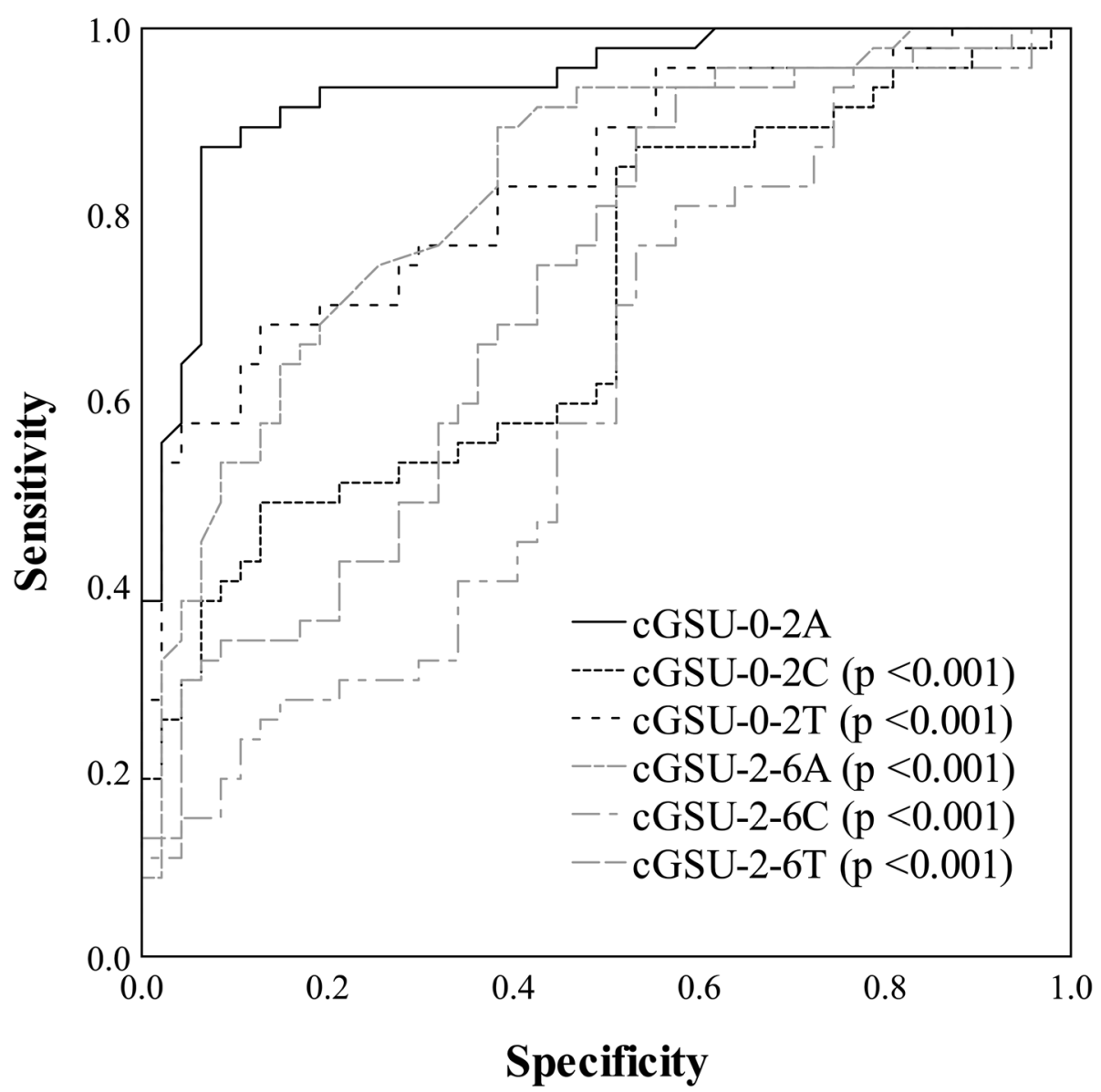

Fig. 1 Receiver operator characteristic (ROC) curve analysis of densitometry data in different regions. ROC analysis determined corrected densitometry data in anterior layer of $0-2 \mathrm{~mm}$ ring (CGSU-0-2A) as the most sensitive parameter of corneal densitometry changes after crosslinking (CXL). All significantly increased corrected densitometry data at 12 months from the central rings mostly affected by CXL were plotted, i.e. anterior, center and total layers of 0-2 mm and of 2-6 mm rings (cGSU-0-2A, cGSU-0-2C, cGSU-0-2T and cGSU-2-6A, cGSU-2-6C, cGSU-2-6T). Comparison of AUCs showed AUC of CGSU-0-2A was statistically significant compared to other AUCs ( $p$-values are shown for corresponding parameters)

three central rings $(0-2 \mathrm{~mm}, 2-6 \mathrm{~mm}, 6-10 \mathrm{~mm})$. Densitometry changes were restricted to the anterior and center layers, while the posterior layer was not affected in any region. These findings supported our expectations, since cross-linking has its predominant effect within $300 \mu \mathrm{m}$ depth in corneal stroma [23, 24]. According to some previous clinical trials $[25,26]$ corneal haze rarely lasts longer than 12 months; however, in these studies haze assessment was performed by slit-lamp examination. Grading of postoperative haze was first described by Fantes et al. based on slit-lamp observation of the cornea and rating haziness on a scale from 0 to 4 [27]. Using slit-lamp evaluation, it is generally difficult to detect very subtle changes in haze levels, thus this scoring system for the purpose of statistical analysis is not refined enough. Moreover, grading on this scale is a subjective method with an inherent lack of intra- and interobserver repeatability and reproducibilty [28].
Scheimpflug-camera with add-on densitmetry software gives the possibility of a more sensitive and reproducible method of corneal haze detection. Our results showed that one year after CXL, densitometry values remained elevated in the anterior, center and total layers of 0-2 $\mathrm{mm}$ and of 2-6 $\mathrm{mm}$ rings, and in the center and total layer of 6-10 $\mathrm{mm}$ ring compared to the baseline. This pattern is very similar to those studies in which densitometry changes were detected by Scheimpflug imaging [12-15], and suggest that cellular modifications in the central cornea persist at least one year after treatment.

In the literature there are contradictory data on which corneal region is mostly affected by CXL. Most of the studies describe that the highest densitometry change can be measured in the anterior layer $[12,13]$, while the main involvement of the center layer has also been reported [15]. It might be because the thickness of center corneal layer is not defined in exact $\mu \mathrm{m}$ in Cornea 


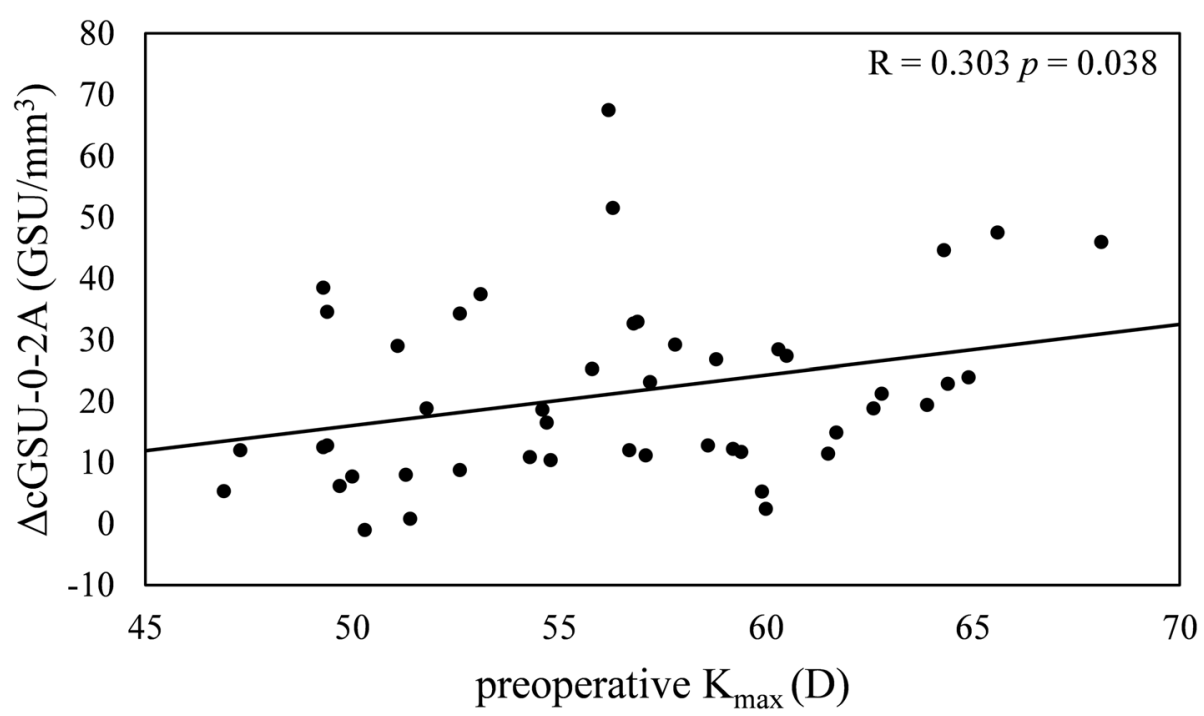

Fig. 2 Relationship between the changes in haze $\left(\Delta \mathrm{cGSU}-0-2 \mathrm{~A}, \mathrm{GSU} / \mathrm{mm}^{3}\right)$ and preoperative maximum keratometry $\left(K_{\max }, \mathrm{D}\right)$. Statistically significant correlation was found between $\Delta$ CGSU-0-2A and preoperative $K_{\max }$

Densito software of Pentacam HR, and differs in every individual. Therefore, in thicker corneas, the results of center layer represent values measured in a thicker center layer, which may affect the final analysis. Moreover, examined rings have different surface areas, which may influence the obtained results [13]. In our study, we used a new additional calculation for densitometry data decribed by Nemeth et al. [13], with which we got surface area- and thickness-corrected densitometry values. Using surface area- and thickness corrected densitometry values allows values to be evaluated independently of the individual corneal thickness or different surface areas of examined rings. With analysing densitometry using corrected data, our study showed that densitometry alteration in anterior layer of $0-2 \mathrm{~mm}$ ring proved to be the most relevant parameter of corneal densitometry changes.

Most of the previous studies investigated the correlations between CXL-induced haze and postoperative outcome $[12,15,16]$, while the predictive impact of preoperative parameteres on postoperative degree of haze after conventional CXL has not been defined so far.

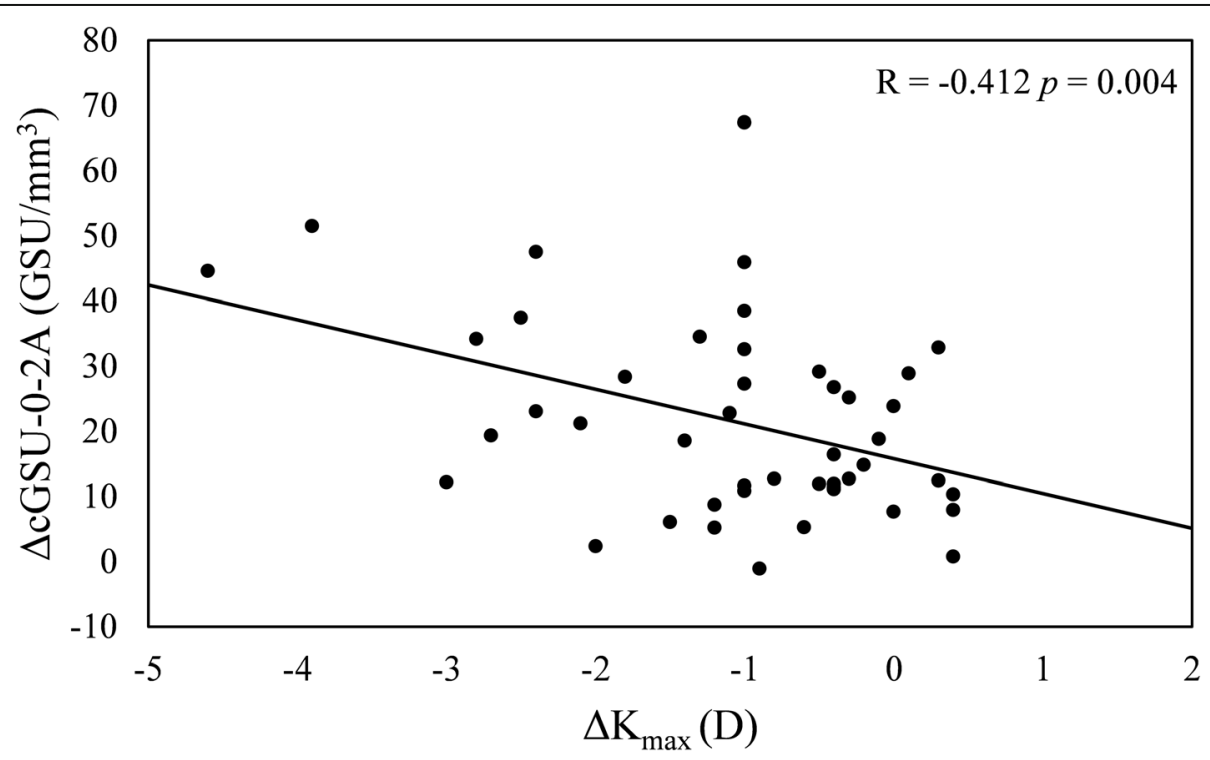

Fig. 3 Relationship between changes in densitometry at 12 months ( $\Delta$ cGSU-0-2A, GSU/mm ${ }^{3}$ ) and changes in maximum keratometry $\left(\Delta K_{\text {max }}\right.$ D). Higher decrease in $\mathrm{K}_{\max }$ (i.e. corneal flattening) is associated with greater increase of densitometry 


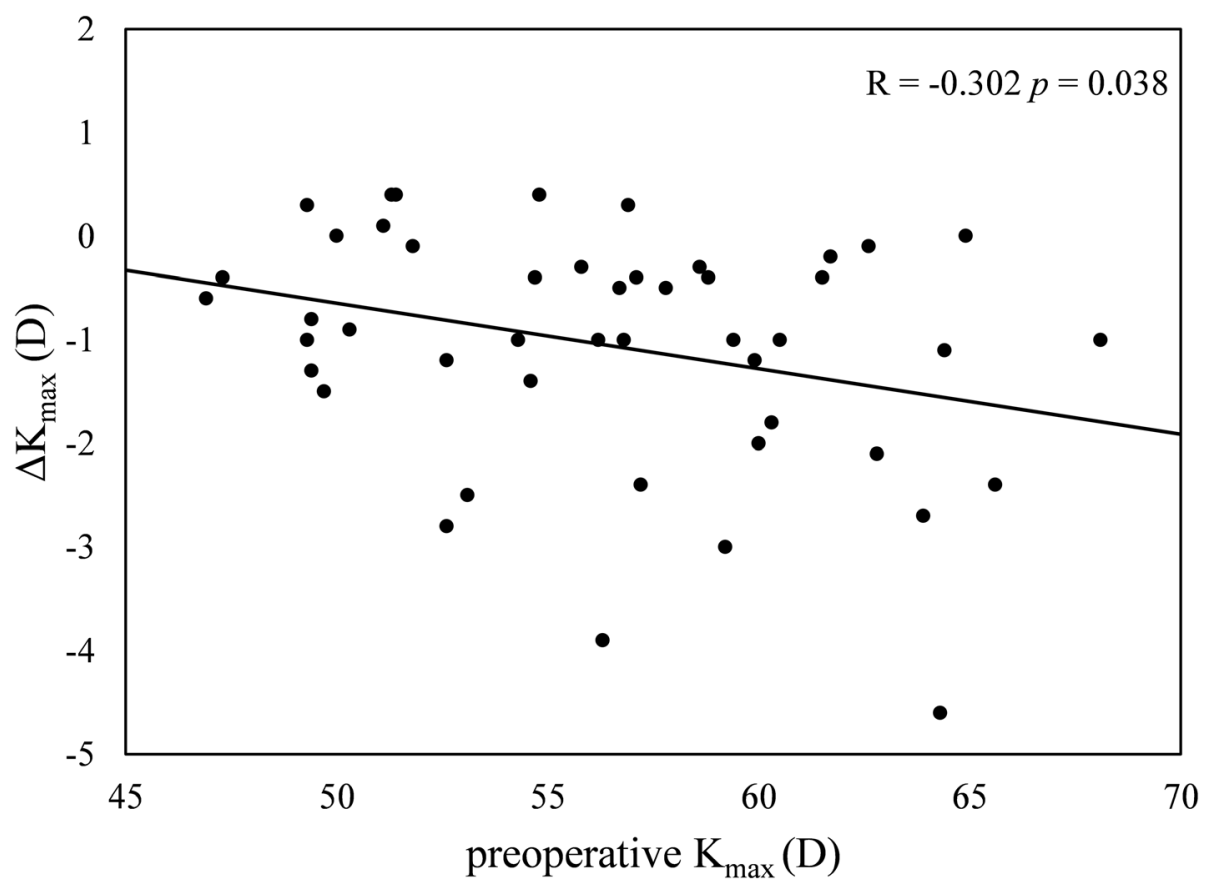

Fig. 4 Changes in maximum keratometry $\left(\Delta K_{\max }, D\right)$ significantly correlated with preoperative maximum keratometry $\left(K_{\max }, D\right)$

Pircher et al. [12] reported significantly higher densitometry values in eyes with greater decrease in keratometry readings, but the exact correlation has not been revealed. In our study, authors analysed the influence of relevant preoperative parameters defining the stage of keratoconus, i.e. ThCT and $\mathrm{K}_{\max }$ on haze formation and corneal flattening. Preoperative $K_{\max }$ was the only preoperative factor which correlated significantly with changes in haze and with changes in keratometry. Recently, several studies [29-31] have highlighted the prognostic significance of preoperative keratometry in determining the amount of topographic flattening after CXL. Our study has also demonstrated that a greater flattening might be expected in corneas with higher preoperative keratomtery readings. Ultrastructural findings of keratoconic corneas include high portion of loosely packed and randomly oriented collagen fibrils [32]. Extent of structural alterations varies as the disease progresses [33]. In case

Table 3 Results of generalized estimating equations adjusted for preoperative $K_{\max }$ readings to determine relationship between postoperative corneal haze (cGSU-0-2 A, GSU/mm³) and postoperative uncorrected and best corrected distance visual acuity (UCDVA and BCDVA, logMAR)

\begin{tabular}{lcrrcrr}
\hline & UCDVA & & & \multicolumn{2}{c}{ BCDVA } \\
\cline { 2 - 3 } & $\boldsymbol{\beta}$ coefficient & $\boldsymbol{p}$-value & & $\boldsymbol{\beta}$ coefficient & $\boldsymbol{p}$-value \\
\hline CGSU-0-2A & 0.006 & 0.041 & & 0.003 & 0.039 \\
$K_{\max }$ & 0.026 & $<0.001$ & & 0.018 & $<0.001$ \\
\hline
\end{tabular}

of advanced keratoconus, cross-linking effect may penetrate relatively deeper, thus, larger proportion of stroma becomes crosslinked, which may result in greater amount of corneal haze. This could be a reason for more pronounced haze formation after CXL in patients with high keratometry readings and suggests that increased densitometry values might be predictive of effectiveness of CXL in terms of corneal flattening and stiffening.

Patients' age might have an effect on the densitometry values determined by Pentacam in normal corneas [34]. It also has been described that the corneal clarity depends on the severity of keratoconus: in more advanced keratoconus, due to the increasing corneal damage, higher densitometry values can be measured [35]. Studies examining corneal densitometry changes after CXL in keratoconus differ in involved patients' ages: some of these reports strictly contain adults over 18 years [12, $15,36,37]$, whereas some of them involved patients under 18 years as well $[11,13,38]$, although the presented tendencies in densitometry changes are similar. A recent study showed that in juvenile keratoconus patients significantly higher postoperative haziness can be observed after CXL compared to adults [39]. Our results showed that the postoperative densitometry changes after CXL depend rather on the keratoconus severity, than on the patients' age, which is in consistent with the available literature $[12,15,16,35]$.

Numerous studies have investigated the visual outcome after conventional cross-linking and reported results are contradictory. Some authors have reported 
significant improvement of BCDVA [15, 40, 41], while others did not support that $[12,14,31]$. According to some recently available data [42-44], preoperative keratometry has a significant impact on postoperative outcome in terms of visual acuity, i.e. patients with higher preoperative keratometry values are likely to show more improvement in BCDVA. The results of several previous researches suggest that CXL-associated stromal haze does not affect high-contrast visual acuity [12, 14]. In our study, an analysis was performed how UCDVA and BCDVA at 12 months would alter with a one-unit change in densitometry values. Therefore, the impact of densitometry values on UCDVA and BCDVA were assessed via generalized estimating equations. Preoperative keratometry readings were kept in the model to adjust its impact on visual outcome. Using this model, we found that elevated densitometry values negatively affected visual acuity one year after treatment. In our study, improvement was not significant neither in UCDVA nor in BCDVA at the end of follow-up period, although significant corneal flattening was observed. According to our results, it can be concluded that in spite of the fact that CXL treatment decreases keratometry values and may improve vision, loss of transparency may have a limiting effect on improvement of visual acuity.

Currently, there is no generally accepted protocol for the type of postoperatively used topical corticosteroids after CXL. Recent literature suggests that fluorometholone is a commonly used topical corticosteroid after CXL for preventing or minimizing corneal haze formation $[13,16,25,45]$. However, fluorometholone is a low potent corticosteroid and may increase the risk of corneal haze compared to more potent corticosteroids, e.g. dexamethasone or betamethasone. It has been shown that keratoconic eyes may have predisposition to the development of steroid-induced ocular hypertension using topical dexamethasone after CXL [46]. Although there is no data about changes of intraocular pressure during using less potent steroids after CXL. Theoretically, in more advanced keratoconus, where more significant haze formation may be expected, using of potent topical corticosteroids might be considered, but only with close monitoring of eye pressure. Future studies are required in order to reveal the effects of different topical cortiocosteroids on haze formation after CXL.

The findings of this study reported herein have to be seen in the light of some limitations. First, this was a retrospective study with limited number of patients. Second, the follow-up period was one year, although it can not be ruled out that the degree of corneal haze may change thereafter. These limitations may impact the generalizability of our results to greater population of patients with keratoconus underwent CXL and to those, whom several years have elapsed since the treatment.
Some disadvantages of densitometry measurement with Scheimpflug camera must also be mentioned. It has been proven before that the repeatability of densitometry measurement was low in CXL-treated corneas [47], thus, for more exact results, it would have been preferable to use the average value of three measurements at every follow-up time points. Variances in white-to-white distance among patients may lead to the false inclusion of limbus and sclera, thus the presentation of higher densitometry values in peripheral rings [35]. Despite the fact that the instrument provides an objective method for measuring haze, the necessity for such a refined measurement in clinical practice is debatable. Moreover, Cornea Densito software is not available on every Pentacam device since it needs to be installed additionally. Finally, analysing data of separated groups according to the severity of keratoconus migh have influenced our final results. Estimating possible densitometry changes according to different stages based on a detailed classification system (such as $\mathrm{ABCD}$ grading system) might be useful for clinicians in predicting postoperative results as well. Future studies may investigate postoperative densitometry changes in exactly defined keratoconus stages.

\section{Conclusions}

In conclusion, with densitometry modul of Pentacam HR, structural corneal changes after conventional crosslinking can be observed even one year after therapy. Our results demonstrated that more pronounced reduction in maximum keratometry values is associated with a greater increase in corneal densitometry and greater flattening effect. Although cross-linking is effective in stabilizing corneal ectasia and reducing keratometry, loss of transparency may limit the improvement of visual acuity.

\section{Abbreviations}

CXL: Cross-linking; UCDVA: Uncorrected distance visual acuity; BCDVA: Best corrected distance visual aquity; SEQ: Sperical equivalent; ThCT: Thinnest corneal thickness; $K_{\text {max }}$ Maximum keratometry; $K_{\text {mean: }}$ Mean keratometry; GSU: Grey scale unit; cGSU: Corrected densitometry values; ROC: Receiever operating characteristic; AUC: Area under curve; GEE: Generalized estimating equations; $\Delta K_{\max }$ : Changes in $K_{\max }$ value; CGSU-0-2A: Corrected densitometry in the anterior layer of the $0-2 \mathrm{~mm}$ ring; $\Delta$ CGSU-0-2A: Changes in corrected densitometry in the anterior layer of the $0-2 \mathrm{~mm}$ ring; $\Delta$ ThCT: Changes in thinnest pachymetry

\section{Acknowledgements}

The authors are grateful to Gabor Nemeth for detailed explanation of correction of densitometry values.

\section{Authors' contributions}

ACs: organized ethical approval, reviewed the literature, collected data and wrote the manuscript. KK: participated in study desing, performed the statistical analysis, critical reading of the manuscript. PD: performed the statistical analysis, collected data. APS: helped to carry out measurements. HK, ISZ: helped in formatting and language. ZZsN: contributed to the critical reading of the manuscript and provided equipment and facilities. All authors read and approved the final manuscript. 


\section{Authors' information}

ACs is an ophthalmologist with her main field of interest including medical cornea and corneal imaging.

\section{Funding}

This research received no funding from the public, commercial, or not-forprofit sectors.

\section{Availability of data and materials}

The datasets used and/or analysed during the current study are available from the corresponding author on reasonable request.

\section{Declarations}

\section{Ethics approval and consent to participate}

This study was carried out with the approval of the Semmelweis University Regional and Institutional Committee of Sciences and Research Ethics, and followed the tenets of Declaration of Helsinki. This was a retrospective study, hence, informed consent was not required.

\section{Consent for publication}

Not applicable.

\section{Competing interests}

The authors declare that they have no financial interests to declare related to the study.

\section{Author details}

${ }^{1}$ Department of Ophthalmology, Semmelweis University, Budapest, Hungary. 2Department of Clinical Ophthalmology, Semmelweis University, Budapest, Hungary.

Received: 14 May 2020 Accepted: 10 August 2021

Published online: 23 August 2021

\section{References}

1. Mas Tur V, MacGregor C, Jayaswal R, O'Brart D, Maycock N. A review of keratoconus: Diagnosis, pathophysiology, and genetics. Surv Ophthalmol. 2017;62(6):770-83.

2. Dias J, Diakonis VF, Kankariya VP, Yoo SH, Ziebarth NM. Anterior and posterior corneal stroma elasticity after corneal collagen crosslinking treatment. Exp Eye Res. 2013;116:58-62.

3. Wollensak G, Spoerl E, Seiler T. Riboflavin/ultraviolet-a-induced collagen crosslinking for the treatment of keratoconus. Am J Ophthalmol. 2003; 135(5):620-7.

4. Caporossi A, Mazzotta C, Baiocchi S, Caporossi T. Long-term results of riboflavin ultraviolet a corneal collagen cross-linking for keratoconus in Italy: the Siena eye cross study. Am J Ophthalmol. 2010;149(4):585-93.

5. Kymionis GD, Grentzelos MA, Liakopoulos DA, Paraskevopoulos TA, Klados $\mathrm{NE}$, Tsoulnaras KI, Kankariya VP, Pallikaris IG. Long-term follow-up of corneal collagen cross-linking for keratoconus-the Cretan study. Cornea. 2014; 33(10):1071-9.

6. O'Brart DP, Kwong TQ, Patel P, McDonald RJ, O'Brart NA. Long-term followup of riboflavin/ultraviolet A $(370 \mathrm{~nm})$ corneal collagen cross-linking to halt the progression of keratoconus. Br J Ophthalmol. 2013;97(4):433-7.

7. Raiskup F, Theuring A, Pillunat LE, Spoerl E. Corneal collagen crosslinking with riboflavin and ultraviolet-A light in progressive keratoconus: ten-year results. J Cataract Refract Surg. 2015;41(1):41-6.

8. O'Brart DP, Patel P, Lascaratos G, Wagh VK, Tam C, Lee J, O'Brart NA. Corneal Cross-linking to Halt the Progression of Keratoconus and Corneal Ectasia: Seven-Year Follow-up. Am J Ophthalmol. 2015;160(6):1154-63.

9. Spoerl E, Hoyer A, Pillunat LE, Raiskup F. Corneal cross-linking and safety issues. Open Ophthalmol J. 2011;5:14-6.

10. Spadea L, Maraone G, Verboschi F, Vingolo EM, Tognetto D. Effect of corneal light scatter on vision: a review of the literature. Int J Ophthalmol. 2016:9(3):459-64.

11. Mahdavi Fard A, Daei Sorkhabi R, Khazaei M, Nader ND. The effects of collagen cross-linking on corneal density: a comparison between accelerated and conventional methods. Int Ophthalmol. 2019;39(7):1559-66.
12. Pircher N, Pachala M, Prager F, Pieh S, Schmidinger G. Changes in straylight and densitometry values after corneal collagen crosslinking. J Cataract Refract Surg. 2015;41(5):1038-43.

13. Nemeth G, Hassan J, Modis L Jr, Hassan Z. Long-Term Changes in Backscattered Light Measurements in Keratoconus Corneas Treated with Collagen Cross-Linking. Curr Eye Res. 2018;43(1):18-26.

14. Kim BZ, Jordan CA, McGhee CN, Patel DV. Natural history of corneal haze after corneal collagen crosslinking in keratoconus using Scheimpflug analysis. J Cataract Refract Surg. 2016;42(7):1053-9.

15. Mathews PM, De Rojas JO, Rapuano PB, Zemsky CJ, Florakis GJ, Trokel SL, Suh LH. Correlation of Scheimpflug densitometry changes with clinical outcomes after corneal crosslinking. J Cataract Refract Surg. 2018;44(8):9931002.

16. Huang J, Shen Y, Jian W, Xu H, Li M, Zhao J, Zhou X, Liao H. Two-year topographic and densitometric outcomes of accelerated $(45 \mathrm{~mW} / \mathrm{cm}(2))$ transepithelial corneal cross-linking for keratoconus: a case-control study. BMC Ophthalmol. 2018;18(1):337.

17. Kranitz K, Kovacs I, Mihaltz K, Sandor GL, Knorz MC, Nemeth J, Nagy ZZ. Corneal changes in progressive keratoconus after cross-linking assessed by Scheimpflug camera. J Refract Surg. 2012;28(9):645-9.

18. DeLong ER, DeLong DM, Clarke-Pearson DL. Comparing the areas under two or more correlated receiver operating characteristic curves: a nonparametric approach. Biometrics. 1988;44(3):837-45.

19. Naderan M, Shoar S, Kamaleddin MA, Rajabi MT, Naderan M, Khodadadi M. Keratoconus Clinical Findings According to Different Classifications. Cornea. 2015;34(9):1005-11.

20. Qazi Y, Wong G, Monson B, Stringham J, Ambati BK. Corneal transparency: genesis, maintenance and dysfunction. Brain Res Bull. 2010;81(2-3):198-210.

21. Mazzotta C, Balestrazzi A, Baiocchi S, Traversi C, Caporossi A. Stromal haze after combined riboflavin-UVA corneal collagen cross-linking in keratoconus: in vivo confocal microscopic evaluation. Clin Exp Ophthalmol. 2007;35(6): $580-2$.

22. Mazzotta C, Hafezi F, Kymionis G, Caragiuli S, Jacob S, Traversi C, Barabino S, Randleman JB. In Vivo Confocal Microscopy after Corneal Collagen Crosslinking. Ocul Surf. 2015;13(4):298-314.

23. Yam JC, Chan CW, Cheng AC. Corneal collagen cross-linking demarcation line depth assessed by Visante OCT After CXL for keratoconus and corneal ectasia. J Refract Surg. 2012;28(7):475-81.

24. Ng AL, Chan TC, Cheng AC. Conventional versus accelerated corneal collagen cross-linking in the treatment of keratoconus. Clin Exp Ophthalmol. 2016:44(1):8-14.

25. Koller T, Mrochen M, Seiler T. Complication and failure rates after corneal crosslinking. J Cataract Refract Surg. 2009;35(8):1358-62.

26. Wittig-Silva C, Chan E, Islam FM, Wu T, Whiting M, Snibson GR. A randomized, controlled trial of corneal collagen cross-linking in progressive keratoconus: three-year results. Ophthalmology. 2014;121(4):812-21.

27. Fantes FE, Hanna KD, Waring GO 3rd, Pouliquen Y, Thompson KP, Savoldelli M. Wound healing after excimer laser keratomileusis (photorefractive keratectomy) in monkeys. Arch Ophthalmol. 1990;108(5):665-75.

28. Takacs Al, Mihaltz K, Nagy ZZ. Corneal density with the Pentacam after photorefractive keratectomy. J Refract Surg. 2011:27(4):269-77.

29. Koller T, Pajic B, Vinciguerra P, Seiler T. Flattening of the cornea after collagen crosslinking for keratoconus. J Cataract Refract Surg. 2011;37(8): 1488-92.

30. Sloot F, Soeters N, van der Valk R, Tahzib NG. Effective corneal collagen crosslinking in advanced cases of progressive keratoconus. J Cataract Refract Surg. 2013;39(8):1141-5.

31. Kasai K, Kato N, Konomi K, Shinzawa M, Shimazaki J. Flattening effect of corneal cross-linking depends on the preoperative severity of keratoconus. Medicine. 2017;96(40):e8160.

32. Sawaguchi S, Fukuchi T, Abe H, Kaiya T, Sugar J, Yue BY. Three-dimensional scanning electron microscopic study of keratoconus corneas. Arch Ophthalmol. 1998;116(1):62-8.

33. Hayes S, Boote C, Tuft SJ, Quantock AJ, Meek KM. A study of corneal thickness, shape and collagen organisation in keratoconus using videokeratography and X-ray scattering techniques. Exp Eye Res. 2007;84(3):423-34.

34. Garzon N, Poyales F, Illarramendi I, Mendicute J, Janez O, Caro P, Lopez A, Argueso F. Corneal densitometry and its correlation with age, pachymetry, corneal curvature, and refraction. Int Ophthalmol. 2017;37(6):1263-8.

35. Lopes B, Ramos I, Ambrosio R. Jr. Corneal densitometry in keratoconus. Cornea. 2014;33(12):1282-6. 
36. Baksoellah Z, Lavy I, Baydoun L, Hooijmaijers HCM, van Dijk K, Melles GRJ. Corneal Tomographic Changes After UV Cross-Linking for Corneal Ectasia (1Year Results). Cornea. 2017;36(12):1498-502.

37. Bohm M, Shajari M, Remy M, Kohnen T. Corneal densitometry after accelerated corneal collagen cross-linking in progressive keratoconus. Int Ophthalmol. 2019;39(4):765-75.

38. Greenstein SA, Fry KL, Bhatt J, Hersh PS. Natural history of corneal haze after collagen crosslinking for keratoconus and corneal ectasia: Scheimpflug and biomicroscopic analysis. J Cataract Refract Surg. 2010;36(12):2105-14.

39. Alzahrani K, Mofty H, Lin EY, Carley F, Brahma A, Morley D, Biswas S, Hillarby MC. Corneal Imaging and Densitometry Measurements in Juvenile and Adult Keratoconus Patients to Evaluate Disease Progression and Treatment Effects After Corneal Cross-Linking. Clin Optom (Auckl). 2019;11:173-80.

40. Viswanathan D, Males J. Prospective longitudinal study of corneal collagen cross-linking in progressive keratoconus. Clin Exp Ophthalmol. 2013;41(6): 531-6.

41. Toprak I, Yaylali V, Yildirim C. Factors affecting outcomes of corneal collagen crosslinking treatment. Eye (Lond). 2014;28(1):41-6.

42. Badawi AE, Abou Samra WA, El Ghafar AA. Predictive Factors of the Standard Cross-linking Outcomes in Adult Keratoconus: One-Year FollowUp. J Ophthalmol. 2017;2017:4109208.

43. Kirgiz A, Atalay K, Cabuk KS, Kaldirim H, Taskapili M. Factors affecting visual acuity after accelerated crosslinking in patients with progressive keratoconus. Arq Bras Oftalmol. 2016;79(3):151-4.

44. Greenstein SA, Hersh PS. Characteristics influencing outcomes of corneal collagen crosslinking for keratoconus and ectasia: implications for patient selection. J Cataract Refract Surg. 2013;39(8):1133-40.

45. Gutierrez R, Lopez I, Villa-Collar C, Gonzalez-Meijome JM. Corneal transparency after cross-linking for keratoconus: 1-year follow-up. J Refract Surg. 2012;28(11):781-6.

46. Kanellopoulos AJ, Cruz EM, Ang RE, Asimellis G. Higher incidence of steroidinduced ocular hypertension in keratoconus. Eye Vis (Lond). 2016;3:4.

47. Pahuja N, Shetty R, Subbiah P, Nagaraja H, Nuijts RM, Jayadev C. Corneal Densitometry: Repeatability in Eyes With Keratoconus and Postcollagen Cross-Linking. Cornea. 2016;35(6):833-7.

\section{Publisher's Note}

Springer Nature remains neutral with regard to jurisdictional claims in published maps and institutional affiliations.

Ready to submit your research? Choose BMC and benefit from:

- fast, convenient online submission

- thorough peer review by experienced researchers in your field

- rapid publication on acceptance

- support for research data, including large and complex data types

- gold Open Access which fosters wider collaboration and increased citations

- maximum visibility for your research: over $100 \mathrm{M}$ website views per year

At BMC, research is always in progress.

Learn more biomedcentral.com/submissions 